\title{
Elektrische Zahnbürste mit Durchblick
}

Mit der neuen Oral-B GENIUS geht das Unternehmen den nächsten Schritt, um Putzgewohnheiten und damit die PatientenCompliance nachhaltig zu verbessern. Erstmals bietet eine elektrische Zahnbürste in Kombination mit der neuen Oral-B App 4.1 die Möglichkeit der Positionserkennung und eröffnet so eine neue Ära in der interaktiven Zahnpflege. Dank dieser Erkennungs-Technologie können Nutzer nicht nur in Echtzeit verfolgen, ob alle Bereiche im Mund gleichmäßig geputzt oder einzelne Bereiche ausgespart wurden. Das Datenmaterial kann darüber hinaus direkt in der App gespeichert, digital an den Zahnarzt übermittelt und im Rahmen eines persönlichen Praxistermins besprochen werden. Für noch mehr individuellen Putz-Spaß sorgen zudem die 12 verschiedenfarbigen LED-Lichter des SmartRings, die die Zahnbürste in der persönlichen Lieblingsfarbe aufleuchten lassen. Ein

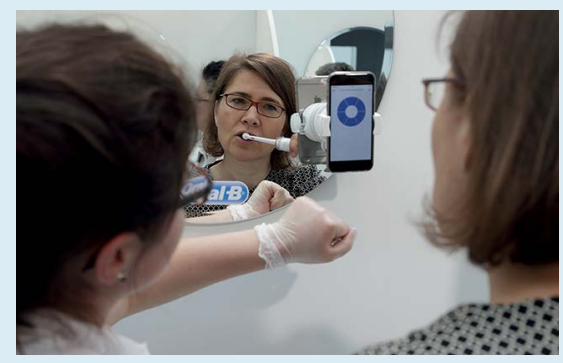

neues Premium Lade-Reise-Etui ermöglicht nicht nur die Aufbewahrung, sondern auch das Aufladen der Bürste sowie des Smartphones durch einen USB-Anschluss. Zusätzlich kann das Smartphone mittels einer separaten Haltevorrichtung z.B. am Spiegel angebracht werden. Ein neuer Lithium-Ionen Akku erlaubt mindestens 12 Tage Putzen (2mal täglich $2 \mathrm{~min}$ ) bis zum nächsten Ladevorgang.

Nach einer Pressemitteilung der Procter \& Gamble GmbH, Schwalbach am Taunus 